\title{
Designing problems for Problem-Based Learning (PBL) sessions: students and faculty perceptions
}

\author{
Anand Kukkamalla ${ }^{1}$, Shobha Karabylu Lakshminarayana ${ }^{1}$, \\ Jessica D'Souza ${ }^{1}$, Shyamala Hande ${ }^{2}$
}

\section{Introduction}

Problem-based learning (PBL) an innovative student centered pedagogic strategy encourages learning through enquiry and exploration. It is an effective tool to cultivate students' ability to learn actively and think critically as learning through problem solving is more effective than memory based learning.

A critical factor in the success of PBL is the problem itself involving the use of complex, "real-world" cases as stimulus and framework for learning. Problem generates learning objectives and hence the driving force behind students' learning. Designing an adequate problem is a challenge as ineffective problem design results in failure of the learning process. Therefore, proper meticulous designing of the problem is essential.

\section{Aim and Objectives}

To analyze the effectiveness of the process of planning and designing of problems by faculty members at Melaka Manipal Medical College (MC).

\footnotetext{
${ }^{1}$ Department of Microbiology,

${ }^{2}$ Department of Anatomy,

Melaka Manipal Medical College (Manipal Campus)*, Manipal, Karnataka, INDIA

Corresponding Author:

Anand Kukkamalla

Selection Grade Lecturer in Microbiology

Melaka Manipal Medical College (Manipal Campus),

International Centre for Health Sciences (I.C.H.S.)

Manipal -576104

Karnataka,

INDIA
}

\section{Materials and Methods}

\section{Problem Designing}

The problems were designed at various levels of functioning keeping in mind the student's task. Problems were meticulously designed for each block (system based study) by faculty members teaching that block to ensure that all major learning objectives were covered.

The problems were discussed later with other colleagues and a core team to assess the validity and reliability of the designed problem following the preparation of the final draft.

\section{Methodology}

Thirsty-six faculty members of $1^{\text {st }}$ and $2^{\text {nd }}$ year teaching at Melaka Manipal Medical College (Manipal Campus) and 346 MBBS students (Year I = 124 and Year II = 222) having PBL as an integral part of their curriculum were included in this study.

The problems were administered during PBL sessions which were brainstormed and presented by students. An anonymous feedback was collected from faculty members in the form of a questionnaire (Table 1) after the completion of problem designing to elicit feedback on the process of problem planning and designing.

Immediately after the problem was discussed and presented by students during PBL session, a feedback was collected from students to assess the quality and effectiveness of the designed problems. The measuring instrument was a self-designed questionnaire having 23 (for students) or 24 items (for faculty) with a 5 point Likert's scale consisting of both open and closed ended questions.

The results were tabulated, analyzed and expressed as numbers, percentages and mean \pm SD. Statistical analysis was performed using independent samples T-test for comparing the means of individual items between students and faculty responses. 


\section{Results}

There were 289 (84\%) students who agreed that problems triggered their thinking and curiosity. Problems gave an insight to issues that had to be discussed 282 (83\%), while $210(74 \%)$ provided ample scope to elicit group discussion. Clinically oriented 275 (81\%), was related to real life situations 263 (76\%); were appropriate to the level of understanding the curriculum 272 (79\%) and relevant for future practice 260 (76\%); while the majority 272 (79\%) felt that problems were framed in simple language. However, $230(67 \%)$ focused on and comprehensibility and $210(61 \%)$ on proper construction.

There were 34 (95\%) of faculty members who perceived that problems triggered students' thinking and curiosity. However, 33 (94\%) gave scope to eliciting discussion and 32 (89\%) thought PBL motivated and initiated student's independent learning. Furthermore, $31(86 \%)$ gave an insight into issues and 27 (75\%) probed a deeper understanding of concepts. Regarding problem designing, faculty members believed that problems were clinically oriented 30 (83\%), framed to students' level of understanding 32 (89\%), focused and easily understandable 26 (74\%), emulated sufficient interest 31 (86\%) and had relevance to their future practice 26 (72\%). While 13 (36\%) faculty members felt that there an was integration of pre and para clinical subjects (Table 1).

Independent samples T-test results showed a statistical significance and correlation between the perceptions of students with faculty for items, 1, 2, 5, 8, 9, 15, 16, 19, 20, 21, 22 and 24. (Figure 1)

\section{Discussion}

PBL is said to develop skills in problem solving, self-directed learning. Problems are the driving force for enhancing student active learning as they function as a learning motivator according to Woei Hung (2009). Angeli (2002) stated that selecting and framing effective PBL problems is a daunting and challenging task. PBL problems should strive to induce students to learn higher cognitive functions (Bloom's Taxonomy of Educational Objectives, Bloom - 1956).

In our study, there was an agreement between students and faculty's perceptions. Problem designing was meticulously done aiming to follow guidelines suggested by Buch (1996). The problems were simple, focused, clinically oriented and covered all the learning objectives framed because the most important step is to set goals and objectives as mentioned by Duch (2001). Problems were properly written and scrutinized effectively by a PBL core team. Dolmans et.al (1993) stated that ineffective problems can cause students difficulty in generating learning issues designed by the faculty.

The problems were designed to keep students' level of understanding, related to the real world so that students had a stake in solving the problem. Students' learning could be enhanced by manipulating with the quality of PBL problems because they could influence students' activation of prior knowledge and generation of learning issues as proven by Dolmans (1993). Problems did not have issues that elicited diverse opinions by making learning focused by strengthening group dynamics, rather than encouraging them to work individually as suggested by Duch (2001).

A perfectly designed problem should be one which generated sufficient issues and hypothesis so that learning objectives can be derived. According to Dolmans et al. (1993), effectiveness of problems is related to the congruency between student-generated learning issues and faculty objectives. In our study we have shown that there was a correlation between the ability of students to formulate their learning objectives. 
Table 1: Students \& faculty responses (in numbers \& percentages) on problem designing

\begin{tabular}{|c|c|c|c|c|c|c|c|}
\hline \multirow{2}{*}{ No. } & \multirow{2}{*}{ Parametres } & \multicolumn{3}{|c|}{ Student response } & \multicolumn{3}{|c|}{ Faculty response } \\
\hline & & Agree & $\begin{array}{c}\text { Un- } \\
\text { Certain }\end{array}$ & $\begin{array}{l}\text { Dis- } \\
\text { Agree }\end{array}$ & Agree & $\begin{array}{l}\text { Un- } \\
\text { Certain }\end{array}$ & $\begin{array}{l}\text { Dis- } \\
\text { Agree }\end{array}$ \\
\hline 1. & $\begin{array}{l}\text { Triggers students' thinking and } \\
\text { curiosity }\end{array}$ & $\begin{array}{c}289 \\
(83.8 \%)\end{array}$ & $\begin{array}{c}40 \\
(11.6 \%)\end{array}$ & $\begin{array}{c}16 \\
(4.6 \%)\end{array}$ & $\begin{array}{c}34 \\
(94.4 \%)\end{array}$ & $\begin{array}{c}2 \\
(5.6 \%)\end{array}$ & $\begin{array}{c}0 \\
(0 \%)\end{array}$ \\
\hline 2. & Have repetition of issues & $\begin{array}{c}131 \\
(38.3 \%) \\
\end{array}$ & $\begin{array}{c}133 \\
(38.9 \%) \\
\end{array}$ & $\begin{array}{c}78 \\
(22.8 \%) \\
\end{array}$ & $\begin{array}{c}4 \\
(11.8 \%) \\
\end{array}$ & $\begin{array}{c}14 \\
(41.1 \%) \\
\end{array}$ & $\begin{array}{c}16 \\
(47.1 \%) \\
\end{array}$ \\
\hline 3. & $\begin{array}{l}\text { Give an insight to the issues that } \\
\text { has to be discussed }\end{array}$ & $\begin{array}{c}282 \\
(83.2 \%) \\
\end{array}$ & $\begin{array}{c}44 \\
(13 \%)\end{array}$ & $\begin{array}{c}13 \\
(3.8 \%)\end{array}$ & $\begin{array}{c}31 \\
(86 \%) \\
\end{array}$ & $\begin{array}{c}3 \\
(8.4 \%) \\
\end{array}$ & $\begin{array}{c}2 \\
(5.6 \%) \\
\end{array}$ \\
\hline 4. & $\begin{array}{l}\text { Structurally complete and properly } \\
\text { constructed }\end{array}$ & $\begin{array}{c}210 \\
(60.9 \%) \\
\end{array}$ & $\begin{array}{c}114 \\
(33.1 \%) \\
\end{array}$ & $\begin{array}{c}21 \\
(6 \%) \\
\end{array}$ & $\begin{array}{c}22 \\
(62.9 \%) \\
\end{array}$ & $\begin{array}{c}8 \\
(22.9 \%) \\
\end{array}$ & $\begin{array}{c}5 \\
(14.2 \%) \\
\end{array}$ \\
\hline 5. & Clinically oriented & $\begin{array}{c}275 \\
(80.5 \%) \\
\end{array}$ & $\begin{array}{c}46 \\
(13.5 \%)\end{array}$ & $\begin{array}{c}21 \\
(6 \%)\end{array}$ & $\begin{array}{c}30 \\
(83.4 \%) \\
\end{array}$ & $\begin{array}{c}5 \\
(13.9 \%) \\
\end{array}$ & $\begin{array}{c}1 \\
(2.7 \%) \\
\end{array}$ \\
\hline 6. & $\begin{array}{l}\text { Cover all the learning objectives } \\
\text { framed }\end{array}$ & $\begin{array}{c}218 \\
(63.6 \%)\end{array}$ & $\begin{array}{c}87 \\
(25.4 \%)\end{array}$ & $\begin{array}{c}38 \\
(11 \%)\end{array}$ & $\begin{array}{c}19 \\
(52.8 \%)\end{array}$ & $\begin{array}{c}10 \\
(27.8 \%) \\
\end{array}$ & $\begin{array}{c}7 \\
(19.4 \%) \\
\end{array}$ \\
\hline 7. & Focused and comprehendible & $\begin{array}{c}230 \\
(67.3 \%)\end{array}$ & $\begin{array}{c}98 \\
(28.7 \%)\end{array}$ & $\begin{array}{c}14 \\
(4 \%)\end{array}$ & $\begin{array}{c}26 \\
(74.3 \%)\end{array}$ & $\begin{array}{c}5 \\
(14.3 \%) \\
\end{array}$ & $\begin{array}{c}4 \\
(11.4 \%) \\
\end{array}$ \\
\hline 8. & Gives scope for eliciting discussion & $\begin{array}{c}210 \\
(73.5 \%)\end{array}$ & $\begin{array}{c}54 \\
(18.9 \%)\end{array}$ & $\begin{array}{c}22 \\
(7.6 \%)\end{array}$ & $\begin{array}{c}33 \\
(94.2 \%) \\
\end{array}$ & $\begin{array}{c}2 \\
(5.8 \%) \\
\end{array}$ & $\begin{array}{c}0 \\
(0 \%)\end{array}$ \\
\hline 9. & $\begin{array}{l}\text { Framed logically and in simple } \\
\text { language }\end{array}$ & $\begin{array}{c}272 \\
(78.6 \%) \\
\end{array}$ & $\begin{array}{c}62 \\
(18 \%) \\
\end{array}$ & $\begin{array}{c}12 \\
(3.4 \%) \\
\end{array}$ & $\begin{array}{c}30 \\
(88.2 \%) \\
\end{array}$ & $\begin{array}{c}3 \\
(8.9 \%) \\
\end{array}$ & $\begin{array}{c}1 \\
(2.9 \%) \\
\end{array}$ \\
\hline 10. & More realistic and less complex & $\begin{array}{c}234 \\
(68 \%) \\
\end{array}$ & $\begin{array}{c}83 \\
(24.2 \%) \\
\end{array}$ & $\begin{array}{c}27 \\
(7.8 \%) \\
\end{array}$ & $\begin{array}{c}27 \\
(74.7 \%) \\
\end{array}$ & $\begin{array}{c}6 \\
(16.7 \%) \\
\end{array}$ & $\begin{array}{c}3 \\
(8.4 \%) \\
\end{array}$ \\
\hline 11. & Relate the subject to the real world & $\begin{array}{c}263 \\
(76.2 \%) \\
\end{array}$ & $\begin{array}{c}58 \\
(16.9 \%)\end{array}$ & $\begin{array}{c}24 \\
(6.9 \%) \\
\end{array}$ & $\begin{array}{c}28 \\
(77.8 \%) \\
\end{array}$ & $\begin{array}{c}4 \\
(11.1 \%) \\
\end{array}$ & $\begin{array}{c}4 \\
(11.1 \%) \\
\end{array}$ \\
\hline 12. & $\begin{array}{l}\text { Motivate, focus and initiate } \\
\text { students' learning }\end{array}$ & $\begin{array}{c}233 \\
(67.3 \%) \\
\end{array}$ & $\begin{array}{c}77 \\
(22.3 \%) \\
\end{array}$ & $\begin{array}{c}36 \\
(10.4 \%) \\
\end{array}$ & $\begin{array}{c}32 \\
(88.9 \%) \\
\end{array}$ & $\begin{array}{c}1 \\
(2.8 \%) \\
\end{array}$ & $\begin{array}{c}3 \\
(8.3 \%) \\
\end{array}$ \\
\hline 13. & $\begin{array}{l}\text { Probe deeper understanding of the } \\
\text { concepts }\end{array}$ & $\begin{array}{c}231 \\
(67.5 \%) \\
\end{array}$ & $\begin{array}{c}84 \\
(24.6 \%) \\
\end{array}$ & $\begin{array}{c}27 \\
(7.9 \%) \\
\end{array}$ & $\begin{array}{c}27 \\
(74.9 \%) \\
\end{array}$ & $\begin{array}{c}6 \\
(16.8 \%) \\
\end{array}$ & $\begin{array}{c}3 \\
(8.3 \%) \\
\end{array}$ \\
\hline 14. & $\begin{array}{l}\text { Connect the previously learned } \\
\text { knowledge }\end{array}$ & $\begin{array}{c}205 \\
(59.5 \%)\end{array}$ & $\begin{array}{c}84 \\
(24.4 \%)\end{array}$ & $\begin{array}{c}56 \\
(16.1 \%)\end{array}$ & $\begin{array}{c}17 \\
(47.2 \%)\end{array}$ & $\begin{array}{c}12 \\
(33.4 \%)\end{array}$ & $\begin{array}{c}7 \\
(19.4 \%)\end{array}$ \\
\hline 15. & $\begin{array}{l}\text { Appropriate to the stage of } \\
\text { curriculum and level of } \\
\text { understanding }\end{array}$ & $\begin{array}{c}272 \\
(78.9 \%)\end{array}$ & $\begin{array}{c}55 \\
(15.9 \%)\end{array}$ & $\begin{array}{c}18 \\
(5.2 \%)\end{array}$ & $\begin{array}{c}32 \\
(88.9 \%)\end{array}$ & $\begin{array}{c}2 \\
(5.6 \%)\end{array}$ & $\begin{array}{c}2 \\
(5.5 \%)\end{array}$ \\
\hline 16. & $\begin{array}{l}\text { Emulates sufficient interest for } \\
\text { students }\end{array}$ & $\begin{array}{c}183 \\
(52.9 \%) \\
\end{array}$ & $\begin{array}{c}126 \\
(36.5 \%) \\
\end{array}$ & $\begin{array}{c}37 \\
(10.6 \%) \\
\end{array}$ & $\begin{array}{c}31 \\
(86.1 \%) \\
\end{array}$ & $\begin{array}{c}2 \\
(5.6 \%) \\
\end{array}$ & $\begin{array}{c}3 \\
(8.3 \%) \\
\end{array}$ \\
\hline 17. & Have relevance to future practice & $\begin{array}{c}260 \\
(75.6 \%) \\
\end{array}$ & $\begin{array}{c}65 \\
(18.9 \%) \\
\end{array}$ & $\begin{array}{c}19 \\
(5.5 \%) \\
\end{array}$ & $\begin{array}{c}26 \\
(72.3 \%) \\
\end{array}$ & $\begin{array}{c}6 \\
(16.6 \%) \\
\end{array}$ & $\begin{array}{c}4 \\
(11.1 \%) \\
\end{array}$ \\
\hline 18. & $\begin{array}{l}\text { Have too many clinical } \\
\text { terminologies }\end{array}$ & $\begin{array}{c}74 \\
21.4 \%) \\
\end{array}$ & $\begin{array}{c}99 \\
(28.7 \%) \\
\end{array}$ & $\begin{array}{c}172 \\
(49.9 \%)\end{array}$ & $\begin{array}{c}6 \\
(16.7 \%) \\
\end{array}$ & $\begin{array}{c}7 \\
(19.5 \%) \\
\end{array}$ & $\begin{array}{c}23 \\
(63.8 \%) \\
\end{array}$ \\
\hline 19. & Ambiguous and multidirectional & $\begin{array}{c}119 \\
(34.8 \%)\end{array}$ & $\begin{array}{c}116 \\
(33.9 \%) \\
\end{array}$ & $\begin{array}{c}107 \\
(31.3 \%) \\
\end{array}$ & $\begin{array}{c}5 \\
(13.9 \%) \\
\end{array}$ & $\begin{array}{c}5 \\
(13.9 \%) \\
\end{array}$ & $\begin{array}{c}26 \\
(72.2 \%) \\
\end{array}$ \\
\hline 20. & $\begin{array}{l}\text { Have controversial issues that will } \\
\text { elicit diverse opinions }\end{array}$ & $\begin{array}{c}145 \\
(42.2 \%)\end{array}$ & $\begin{array}{c}86 \\
(25 \%) \\
\end{array}$ & $\begin{array}{c}113 \\
(32.8 \%)\end{array}$ & $\begin{array}{c}5 \\
(13.9 \%)\end{array}$ & $\begin{array}{c}14 \\
(38.9 \%)\end{array}$ & $\begin{array}{c}17 \\
(47.2 \%) \\
\end{array}$ \\
\hline 21. & Too confusing & $\begin{array}{c}53 \\
(15.4 \%)\end{array}$ & $\begin{array}{c}104 \\
(30.3 \%)\end{array}$ & $\begin{array}{c}186 \\
(54.3 \%)\end{array}$ & $\begin{array}{c}1 \\
(2.8 \%) \\
\end{array}$ & $\begin{array}{c}8 \\
(22.3 \%) \\
\end{array}$ & $\begin{array}{c}27 \\
(74.9 \%)\end{array}$ \\
\hline 22. & Too lengthy & $\begin{array}{c}70 \\
(20.2 \%) \\
\end{array}$ & $\begin{array}{c}103 \\
(29.8 \%) \\
\end{array}$ & $\begin{array}{c}173 \\
(50 \%) \\
\end{array}$ & $\begin{array}{c}3 \\
(8.8 \%) \\
\end{array}$ & $\begin{array}{c}5 \\
(14.7 \%) \\
\end{array}$ & $\begin{array}{c}26 \\
(76.5 \%) \\
\end{array}$ \\
\hline 23. & $\begin{array}{l}\text { Designed after discussion with } \\
\text { fellow faculty members }\end{array}$ & NA & NA & NA & $\begin{array}{c}22 \\
(61 \%)\end{array}$ & $\begin{array}{c}8 \\
(22.3 \%) \\
\end{array}$ & $\begin{array}{c}6 \\
(16.7 \%) \\
\end{array}$ \\
\hline 24. & $\begin{array}{l}\text { Designed to accomplish integration } \\
\text { of pre \& para clinical subjects }\end{array}$ & $\begin{array}{c}186 \\
(53.9 \%)\end{array}$ & $\begin{array}{c}123 \\
(35.6 \%)\end{array}$ & $\begin{array}{c}36 \\
(10.5 \%)\end{array}$ & $\begin{array}{c}13 \\
(36.1 \%)\end{array}$ & $\begin{array}{c}6 \\
(16.6 \%)\end{array}$ & $\begin{array}{c}17 \\
(47.3 \%)\end{array}$ \\
\hline
\end{tabular}




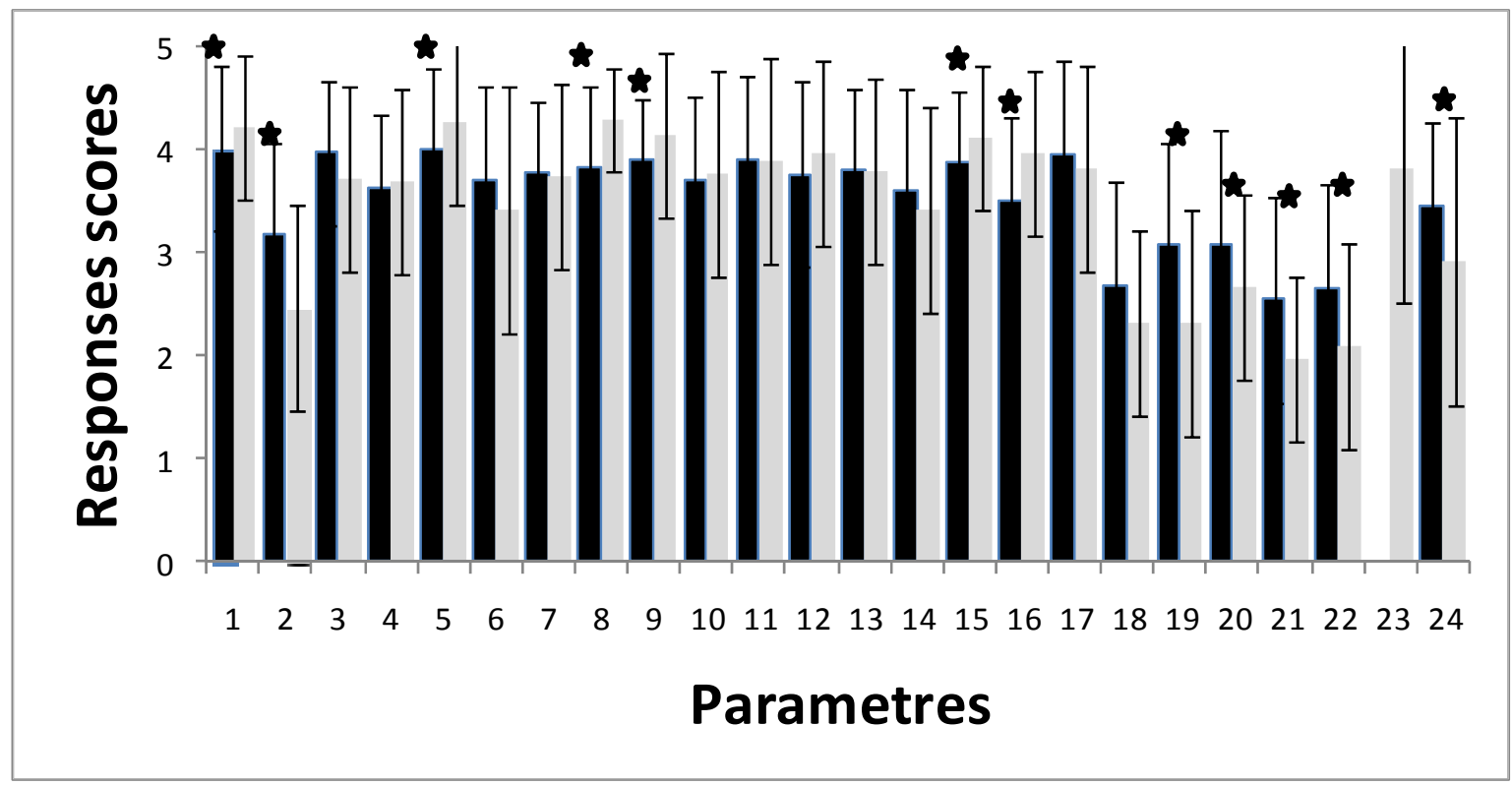

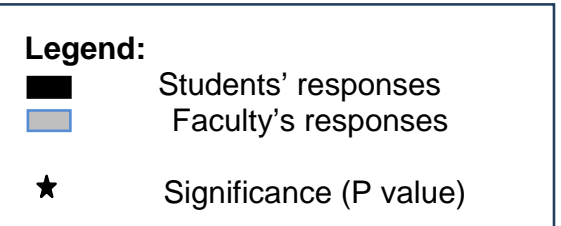

Legend for Parametres
1. Triggers students' thinking and curiosity
2. Have repetition of issues
3. Give an insight to the issues that has to be discussed
4. Structurally complete and properly constructed
5. Clinically oriented
6. Cover all the learning objectives framed
7. Focused and comprehendible
8. Gives scope for eliciting discussion
9. Framed logically and in simple language
10. More realistic and less complex
11. Relate the subject to the real world
12. Motivate, focus and initiate students' learning
13. Probe deeper understanding of the concepts
14. Connect the previously learned knowledge
15. Appropriate to the stage of curriculum and level of
understanding
16. Emulates sufficient interest for students
17. Have relevance to future practice
18. Have too many clinical terminologies
19. Ambiguous and multidirectional
20. Have controversial issues that will elicit diverse opinions
21. Too confusing
22. Too lengthy
23. Designed after discussion with fellow faculty members
24. Designed to accomplish integration of pre \& para clinical
subjects




\section{Conclusion}

The process of problem designing and problems functioned as effective triggers to initiate self-directed learning, stimulated thinking making PBL sessions interesting. It brought out the learning objectives, encouraged discussion, stimulated concept understanding and critical thinking. These problems should be revised regularly after feedback. There was considerable significant co-relation between the perceptions of student and faculty in terms of problem designing. Problems designed could integrate pre and para clinical subjects paving way for vertical and horizontal integration.

Since only few problems were analyzed during this study, further research is necessary to assess the validity of problems using certain instruments/rubric.

\section{References}

Angeli, C. (2002) Teachers' practical theories for the design and implementation of problembased learning, Sci. Educ.Int. 13, 3, pp.9-15.

Buch, B. (1996) University of Delaware, [online]. Available at: http://www.udel.edu/pbl/cte/spr96phys.html.

Dolmans D. H. J. M., Gijselaers W. H., Schmidt, H. G. \& van der Meer, S.B. (1993) Problem effectiveness in a course using problem-based learning, Acad. Med., 68, 3, pp.207-213.

Duch B. J. (2001) Writing problems for deeper understanding. In: B.J. Duch, S. E. Groh, \& Allen, D. E., ed. (2001) The Power of ProblemBased Learning: A Practical 'How To' for Teaching Undergraduate Courses in Any Discipline. Sterling, VA: Stylus Publishing, pp.47-53.

Duch, B. J., Groh, S. E., \& Allen, D. E., Eds. (2001). The Power of Problem-Based Learning: A Practical 'How to' for Teaching Undergraduate Courses in Any Discipline. Sterling, VA: Stylus Publishing.

Woei Hung. (2009) The 9-step problem design process for problem-based learning: Application of the 3C3R model, Educational Research Review, 4, 2, pp.118-141. 